\title{
Minimum Component Multiple Integrator Loop OTA-Grounded Capacitor All-Pole Filters
}

\author{
Yichuang Sun and J. K. Fidler \\ Department of Electronics, University of York, York YO1 5DD, UK
}

\begin{abstract}
This paper addresses multiple loop feedback minimum component OTA-grounded capacitor all-pole filters. A general theory and its formulation are presented. It is shown that many new interesting configurations can be produced, all popular approximations including the Butterworth and Chebyshev characteristics are realizable and some known typical structures are also derivable from the method. The minimal number of elements, that is, $n$ OTAs and $n$ capacitors for $n$ poles is achieved, all capacitors are grounded, equal-value transconductances and equalvalue capacitances can be arbitrarily selected.
\end{abstract}

\section{INTRODUCTION}

In OTA-C filter design the transconductance gain $g_{m}$ of the OTA is used as a design parameter in the same way as $R$ 's are used in conventional active- $R C$ filters and as a result several advantages have been achieved [1-12]. However, the number of active components in OTA-C filters is rapidly growing as the filter order increases. The larger number of elements generally increases power consumption, the occupied chip area, noise, circuit parasitics and unreliability. It is also well known that grounded capacitors can be implemented on a smaller area than floating ones and they can absorb many capacitive shunt parasitics. Thus reducing the number of elements and utilizing grounded capacitors becomes a major consideration in HF continuous-time OTA-C filter design [2-5, 8-10].

In active-RC filter design there are mainly three methods: cascade, simulation and multiple loop feedback, and each approach has its own special advantages [13]. For high-order OTA-C filter synthesis, the cascade and simulation methods have been widely used. Recently multiple loop feedback structures have also received much attention [5-12].

This paper will explore new minimum component mul tiple integrator loop OTA-grounded capacitor structures of high-order all-pole filters. After a general formulation of the method in section 2, we will introduce some typical general structures in section 3 . The exhaustive enumeration of filter structures for any given order will be then investigated in section 4 . Section 5 will present numerical design examples, followed by conclusions in section 6 .

\section{THEORY AND APPROACH}

Consider the model in Fig.1. It consists of $n$ OTA-C integrators connected in tandem and a feedback network. All capacitors are grounded. Suppose that the feedback network is without any components. Then the model has the minimum number of components, that is, $n$ OTAs and $n$ capacitors for realizing $n$ th-order all-pole filters.

The feedback network in the model can be characterised by the feedback connection matrix $F$ as follows:

$$
V_{f}=F V_{o}
$$

where $V_{o}=\left[\begin{array}{lll}V_{o 1} & V_{o 2} \cdots V_{o n}\end{array}\right]^{t}, V_{f}=\left[\begin{array}{ll}V_{f 1} & V_{f 2} \\ \cdots & V_{f n}\end{array}\right]^{t}$, and $F=\left[f_{i j}\right]_{n \times n}$. The superscript $t$ denotes transpose.

Since there are no components in the feedback network,

$f_{i j}= \begin{cases}1 & \text { if there is a connection between } V_{f i} \text { and } V_{o j} \\ 0 & \text { otherwise }\end{cases}$

Also, in $F$ each row has one and only one unit element, for all $f_{i j}=1$ there are $i \leq j$, and $f_{n n}=1[10,11]$.

With $\tau_{j}=C_{j} / g_{m j}$, the part of the $n$ integrators connnected in tandem can be described by

$$
\begin{gathered}
M(s) V_{o}=I V_{i n}-V_{f} \\
\text { where } M(s)=\left[\begin{array}{cccc}
s \tau_{1} & & & \\
-1 & s \tau_{2} & & \\
& & \ddots & \\
& & -1 & s \tau_{n}
\end{array}\right] \quad I=\left[\begin{array}{c}
1 \\
0 \\
\vdots \\
0
\end{array}\right]
\end{gathered}
$$

For the whole system combining (1) and (2) yields

$$
A(s) V_{o}=I V_{\text {in }} \quad \text { where } A(s)=M(s)+F
$$

Let us use $A_{i j}(s)$ and $|A(s)|$ to represent the cofactors and determinant of $A(s)$. Using the features of $F$ and the structure of $M(s)$, we demonstrate that $A_{1 n}(s)=1$. The overall transfer function and the sensitivity functions can be then derived from (3) [10], given by

$$
\begin{gathered}
H(s)=V_{\text {out }} / V_{\text {in }}=V_{\text {on }} / V_{\text {in }}=1 /|A(s)| \\
S_{\tau_{1}}^{H(s)}=-s \tau_{1} A_{11}(s) /|A(s)|, S_{\tau_{n}}^{H(s)}=-s \tau_{n} A_{n n}(s) /|A(s)|
\end{gathered}
$$




$$
S_{\tau_{j}}^{H(s)}=-s \tau_{j} A_{j n}(s) A_{1 j}(s) /|A(s)| \quad j=2,3, \ldots, n-1
$$

The introduction of $F$ and the relationship between $F$ and the feedback connection are crucial for the unified analysis, synthesis and structure generation. Equation (3) also reveals clearly the filter performance dependence on the structure.

The design procedure for realizing the all-pole function

$$
H_{d}(s)=1 /\left(b_{n} s^{n}+b_{n-1} s^{n-1}+\cdots+b_{1} s+1\right)
$$

can be summarized as follows: 1) generate all filter structures of order $n$ with different feedback connections, 2) select the structure, 3 ) determine the associated $H(s)$ by expanding $|A(s)|, 4)$ derive coefficient matching equations by comparing $H(s)$ with $\left.H_{d}(s), 5\right)$ solve the equations for $\left.\tau_{j}, 6\right)$ compute the values of $C_{j}$ and $g_{m j}$.

The most important thing in the process is to generate filter topologies, which will be further discussed in the preceeding sections. To expand $|A(s)|$ to attain $H(s)$ and to solve the matching equations for $\tau_{j}$ are another two concerns. For many low-order and some special highorder structures, $|A(s)|$ can be easily handled and the design equations may be solved explicitly. However, more often some symbolic analysis techniques may be required to deal with $|A(s)|$ and in most cases nonlinear equation solving approaches such as the Newton-Raphson algorithm may need to be invoked to solve the $\tau_{j}$ equations. It should also be noted that there are $n$ degrees of freedom in deciding $C_{j}$ and $g_{m j}$ from $\tau_{j}$ and this gives much design flexibility. For example, we may set the equal transconductances $g_{m 1}=g_{m 2}=\cdots=g_{m n}=g_{m}$ and calculate $C_{j}=g_{m} \tau_{j}$, or let $C_{1}=C_{2}=\cdots=C_{n}=C$, that is, the identical capacitances and compute $g_{m j}=C / \tau_{j}$.

\section{SOME TYPICAL ARCHITECTURES}

Three typical configurations, that is, the inverse followthe-leader-feedback (IFLF), leapfrog (LF) and cascade are derivable from the general model and can be designed to realize (5) using the above procedure.

If the elements in the last column of $F$ all are unity and the other elements are zero, the circuit has the IFLF structure as shown in Fig. 2 and $A(s)$ becomes

$$
A(s)=\left[\begin{array}{ccccc}
s \tau_{1} & 0 & & 0 & 1 \\
-1 & s \tau_{2} & & 0 & 1 \\
0 & 0 & & s \tau_{n-1} & 1 \\
0 & 0 & & -1 & s \tau_{n}+1
\end{array}\right]
$$

By expansion using the last column we obtain $|A(s)|=$ $\tau_{1} \tau_{2} \cdots \tau_{n} s^{n}+\tau_{1} \tau_{2} \cdots \tau_{n-1} s^{n-1}+\cdots+\tau_{1} \tau_{2} s^{2}+\tau_{1} s+1$

Comparing it with (5) gives the design equations

$$
\prod_{i=1}^{j} \tau_{i}=b_{j} \quad j=1,2, \ldots, n
$$

By simple manipulation of the equations we obtain

$$
\tau_{1}=b_{1}, \quad \tau_{i}=b_{i} / b_{i-1}
$$

Calculating the concerned cofactors of $A(s)$ we attain the sensitivity functions:

$$
S_{\tau_{h}}^{H(s)}=-\left[\sum_{j=h}^{n}\left(\prod_{i=1}^{j} \tau_{i}\right) s^{j}\right] /\left[\sum_{j=1}^{n}\left(\prod_{i=1}^{j} \tau_{i}\right) s^{j}+1\right]
$$

Substituting the design equations results in

$S_{\tau_{h}}^{H(s)}=-\left(\sum_{j=h}^{n} b_{j} s^{j}\right) /\left(\sum_{j=1}^{n} b_{j} s^{j}+1\right) \quad h=1,2, \ldots, n$

The IFLF structure was also obtained by realizing the signal flow graph of the transfer function [12].

For $F$ with $f_{i j}=1, i=1,2, \ldots, n-1, j=i+1$ and $f_{n n}=1$, we have the LF configuration, as shown in Fig.3. Similarly the transfer function, design formulae and sensitivity functions may also be obtained.

The LF configuration derived above is similar to that obtained by Nawrocki [5]. However the realization techniques are different. In [5] the operational simulation approach of passive low-pass LC ladders was implemented and the OTA-C realization needs quite complicated treatment of terminations.

Finally when $n$ is even and $F=\operatorname{diag}\left\{\left[\begin{array}{ll}0 & 1 \\ 0 & 1\end{array}\right]\right\}$, the circuit becomes the cascade of biquadratic sections.

\section{EXHAUSTIVE SEARCH OF STRUCTURES}

In this section we investigate the generation of all possible filter configurations for a given order $n$ according to the features of the feedback matrix $F$. In our discussion $f_{n n}=1$ will be implied for all situations.

It is very easy to verify that there is only one structrure for $n=1$ and only one is practical of two combinations for $n=2$, which corresponds to $f_{11}=f_{12}=1$. This biquad was previously discussed elsewhere $[1,2]$.

When $n=3[10]$, having tried all 6 possibilities of $F$, we have found that there are only three configurations that are realistic: IFLF, LF and cascade.

For the fourth-order filters, noting that $F$ is an upper triangular matrix and with some tedious manipulation of $|A(s)|$ we obtain the general transfer function as

$$
\begin{aligned}
& H(s)=1 /\left\{\left(\tau_{1} \tau_{2} \tau_{3} \tau_{4}\right) s^{4}+\left(\tau_{1} \tau_{2} \tau_{3} f_{44}+\tau_{1} \tau_{2} \tau_{4} f_{33}+\tau_{1} \tau_{3} \tau_{4} f_{22}\right.\right. \\
& \left.+\tau_{2} \tau_{3} \tau_{4} f_{11}\right) s^{3}+\left[\tau_{1} \tau_{2}\left(f_{33} f_{44}+f_{34}\right)+\tau_{1} \tau_{3} f_{22} f_{44}+\tau_{1} \tau_{4}\left(f_{22} f_{33}\right.\right. \\
& \left.\left.+f_{23}\right)+\tau_{2} \tau_{3} f_{11} f_{44}+\tau_{2} \tau_{4} f_{11} f_{33}+\tau_{3} \tau_{4}\left(f_{11} f_{22}+f_{12}\right)\right] s^{2} \\
& +\tau_{1}\left(f_{22} f_{33} f_{44}+f_{22} f_{34}+f_{23} f_{44}+f_{24}\right)+\tau_{2}\left(f_{11} f_{33} f_{44}\right. \\
& \left.+f_{11} f_{34}\right)+\tau_{3}\left(f_{11} f_{22} f_{44}+f_{12} f_{44}\right)+\tau_{4}\left(f_{11} f_{22} f_{33}+f_{11} f_{23}\right. \\
& \left.\left.+f_{12} f_{33}+f_{13}\right)\right] s+\left(f_{11} f_{22} f_{33} f_{44}+f_{11} f_{22} f_{34}+f_{11} f_{44} f_{23}\right. \\
& \left.\left.\quad+f_{12} f_{33} f_{44}+f_{11} f_{24}+f_{13} f_{44}+f_{12} f_{34}+f_{14}\right)\right\}
\end{aligned}
$$

According to the properties of $F$ there are altogether 24 combinations of filter configurations. Among them 10 structures are proved practical and are shown in Fig. 4. The corresponding $F$ s and $|A(s)|$ s are given below. 
Circuit 1 $: f_{12}=f_{22}=f_{34}=1, \quad \tau_{1} \tau_{2} \tau_{3} \tau_{4} s^{4}+\left(\tau_{1} \tau_{2} \tau_{3}+\right.$ $\left.\tau_{1} \tau_{3} \tau_{4}\right) s^{3}+\left(\tau_{1} \tau_{2}+\tau_{1} \tau_{3}+\tau_{3} \tau_{4}\right) s^{2}+\left(\tau_{1}+\tau_{3}\right) s+1$

Circuit 2: $f_{12}=f_{23}=f_{34}=1$

$\tau_{1} \tau_{2} \tau_{3} \tau_{4} s^{4}+\tau_{1} \tau_{2} \tau_{3} s^{3}+\left(\tau_{1} \tau_{2}+{ }_{1} \tau_{4}+\tau_{3} \tau_{4}\right) s^{2}+\left(\tau_{1}+\tau_{3}\right) s+1$

Circuit $3: f_{12}=f_{24}=f_{34}=1$

$\tau_{1} \tau_{2} \tau_{3} \tau_{4} s^{4}+\tau_{1} \tau_{2} \tau_{3} s^{3}+\left(\tau_{1} \tau_{2}+\tau_{3} \tau_{4}\right) s^{2}+\left(\tau_{1}+\tau_{3}\right) s+1$

Circuit $4: f_{13}=f_{22}=f_{34}=1$

$\tau_{1} \tau_{2} \tau_{3} \tau_{4} s^{4}+\left(\tau_{1} \tau_{2} \tau_{3}+\tau_{1} \tau_{3} \tau_{4}\right) s^{3}+\left(\tau_{1} \tau_{2}+\tau_{1} \tau_{3}\right) s^{2}+\left(\tau_{1}+\tau_{4}\right) s+1$

Circuit 5 : $f_{13}=f_{23}=f_{34}=1$

$\tau_{1} \tau_{2} \tau_{3} \tau_{4} s^{4}+\tau_{1} \tau_{2} \tau_{3} s^{3}+\left(\tau_{1} \tau_{2}+\tau_{1} \tau_{4}\right) s^{2}+\left(\tau_{1}+\tau_{4}\right) s+1$

Circuit 6: $f_{13}=f_{24}=f_{34}=1$

$\tau_{1} \tau_{2} \tau_{3} \tau_{4} s^{4}+\tau_{1} \tau_{2} \tau_{3} s^{3}+\tau_{1} \tau_{2} s^{2}+\left(\tau_{1}+\tau_{4}\right) s+1$

Circuit 7 : $f_{14}=f_{22}=f_{34}=1$

$\tau_{1} \tau_{2} \tau_{3} \tau_{4} s^{4}+\left(\tau_{1} \tau_{2} \tau_{3}+\tau_{1} \tau_{3} \tau_{4}\right) s^{3}+\left(\tau_{1} \tau_{2}+\tau_{1} \tau_{3}\right) s^{2}+\tau_{1} s+1$

Circuit 8: $f_{14}=f_{23}=f_{33}=1$

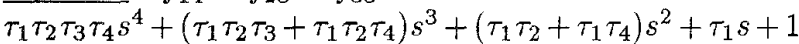

Circuit 9: $f_{14}=f_{23}=f_{34}=1$

$\frac{\tau_{1} \tau_{2} \tau_{3} \tau_{4} s^{4}}{4}+\tau_{1} \tau_{2} \tau_{3} s^{3}+\left(\tau_{1} \tau_{2}+\tau_{1} \tau_{4}\right) s^{2}+\tau_{1} s+1$

Circuit $10: f_{14}=f_{24}=f_{34}=1$

$\tau_{1} \tau_{2} \tau_{3} \tau_{4} s^{4}+\tau_{1} \tau_{2} \tau_{3} s^{3}+\tau_{1} \tau_{2} s^{2}+\tau_{1} s+1$

Note that circuits 1,2 , and 10 [11] are the cascade, LF, and IFLF structures, respectively and the others are new.

Using the method we may systematically generate a large number of all-pole filters. Generally, for the $n$ thorder there are $n$ ! possible configurations, of which three have been discussed in the preceding section.

\section{DESIGN EXAMPLES}

To realize the $4 t h$-order all-pole characteristic (5) the coefficient matching equations of circuits $2,3,5,6,9$ and 10 can be solved explicitly. If the factored form is exploited, the design of circuit 1 can also be tackled analytically.

For the normalized $4 t h$-order Butterworth filter with the inverse transfer function $1 / H_{d}(s)=$

$$
s^{4}+2.61313 s^{3}+3.41421 s^{2}+2.61313 s+1
$$

the parameter values of all the practical structures are calculated from the individual $\tau_{j}$ equations by using the explicit solutions or any nonlinear equation solvers and given in table 1 .

The Chebyshev filters can also be synthesized. For instance, the element values as shown in table 2 are determined for the realization of the $1 d B$ ripple, unity DC gain, frequency-normalized Chebyshev filter with $1 / H_{d}(s)=$

$$
3.62808 s^{4}+3.45688 s^{3}+5.27496 s^{2}+2.69429 s+1
$$

\section{CONCLUSIONS}

The multiple loop feedback all-pole OTA-grounded capacitor filter structures with the minimum number of components have been studied. The proposed approach is systematic and general. A variety of new structures can be generated and designed, and some known filter configurations are simply the special cases of the approach. The generated filter architectures have the fewest components, $n$ OTAs and $n$ capacitors for $n$ th-order unity DC gain all-pole filters. All capacitors are grounded, all transconductances can be identical, and the cut-off frequency is tunable by $g_{m}$.

\section{REFERENCES}

[1] E. Sánchez-Sinencio, R. L. Geiger and H. Nevárez-Lozano, "Generation of continuous-time two integrator loop OTA filter structures," IEEE Trans. Circuits and Syst., Vol.35, No.8, pp.936-946, 1988.

[2] Y. Sun and J. K. Fidler, "Novel OTA-C realizations of biquadratic transfer functions," Int. J. of Electronics, Vol.75, pp.333-348, 1993.

[3] M. A. Tan and R. Schaumann, "A reduction in the number of active components used in transconductance grounded capacitor filters," Proc. IEEE ISCAS'90, pp.2276-2278, 1990.

[4] A. Wyszynski and R. Schaumann, "Using multiple-input transconductors to reduce number of components in OTA-C filter design," Electronics Letters, vol.28, 1992.

[5] R. Nawrocki, "Electronically tunable all-pole low-pass leapfrog ladder filter with operational transconductance amplifier," Int. J. Electronics, Vol.62, No.5, 1987.

[6] R. Nawrocki, "Electronically controlled OTA-C filter with follow- the-leader-feedback structure," Int. J. Cir. Theor. Appl, vol.16, pp.93-96, 1988.

[7] F. Krummenacher, "Design considerations in highfrequency CMOS transconductance amplifier (TAC) filters," Proc. IEEE ISCAS'89, pp.100-105, 1989.

[8] Y. Sun and J. K. Fidler, "Some design methods of OTA-C and CCII-RC filters," IEE 13th Saraga Colloquium on Electronic Filters, pp. 7/1-7/8, 1993

[9] Y. Sun and J. K. Fidler, "OTA-C realization of general high-order transfer functions," Electronics Letters, vol.29, pp.1057-1058, 1993

[10] Y. Sun and J. K. Fidler, "Canonical realization of highorder all-pole low-pass OTA-C filters," Proc. 1993 ECCTD, Part I, pp.69-72, 1993.

[11] Y. Zhang and J. K. Fidler, "A design method for highorder OTA-C low-pass filters with topological structure selection flexibility," Proc. of China ICEMI'g2, 1992.

[12] W. Guo, J. Liu and S. Yang, "The realization of high-order OTA-C filter," Int. J. Electronics, Vol.65, No.6, pp.11531157,1988

[13] R. Schaumann, M. S. Ghausi and K. R. Laker. Design of analog filters: passive, active-RC and switched capacitor. Prentice Hall, Inc., NJ, 1990. 


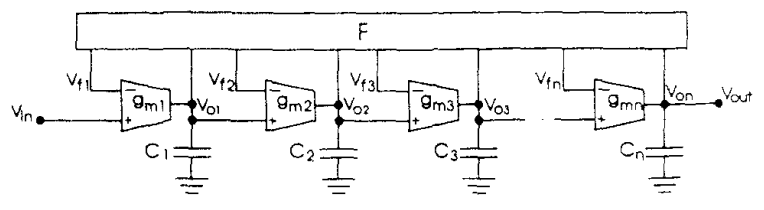

Figure 1: Multiple integrator loop feedback nodel

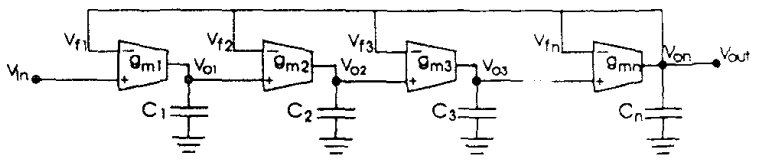

Figure 2: IFLF structure

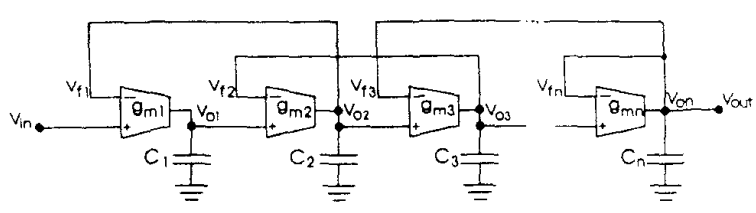

Figure 3: LF configuration

Table 1: Parameter values for Butterworth filter

\begin{tabular}{c|cccc}
\hline Circuit & $\tau_{1}$ & $\tau_{2}$ & $\tau_{3}$ & $\tau_{4}$ \\
\hline 1 & 0.765367 & 1.30656 & 1.84776 & 0.541196 \\
& 1.84776 & 0.541196 & 0.765367 & 1.30656 \\
2 & 1.53073 & 1.57716 & 1.08239 & 0.382683 \\
3 & 1.76763 & 1.74847 & 0.845492 & 0.382683 \\
4 & 1.9453 & 0.896275 & 0.858833 & 0.667826 \\
5 & 2.23044 & 1.14805 & 1.02049 & 0.382683 \\
6 & 2.23044 & 1.53073 & 0.765367 & 0.382683 \\
7 & 2.61313 & 0.667368 & 0.639195 & 0.8971 \\
8 & 2.61313 & 0.639195 & 0.8971 & 0.667368 \\
9 & 2.61313 & 0.92388 & 1.08239 & 0.382683 \\
10 & 2.61313 & 1.30656 & 0.765367 & 0.382683 \\
\hline
\end{tabular}

Table 2: Parameter values for Chebyshev filter

\begin{tabular}{c|cccc}
\hline Circuit & $\tau_{1}$ & $\tau_{2}$ & $\tau_{3}$ & $\tau_{4}$ \\
\hline 1 & 0.28289 & 3.5833 & 2.4114 & 1.48425 \\
& 2.4114 & 1.48425 & 0.28289 & 3.5833 \\
2 & 1.28172 & 1.90934 & 1.41256 & 1.04953 \\
3 & 1.91952 & 2.32445 & 0.774769 & 1.04953 \\
4 & 1.23444 & 3.734 & 0.53917 & 1.45985 \\
5 & 1.64476 & 2.15761 & 0.974114 & 1.04953 \\
6 & 1.64476 & 3.20713 & 0.655337 & 1.04953 \\
7 & 2.69429 & 1.67213 & 0.285702 & 2.81871 \\
8 & 2.69429 & 0.285702 & 2.81871 & 1.67213 \\
9 & 2.69429 & 0.908307 & 1.41256 & 1.04953 \\
10 & 2.69429 & 1.95783 & 0.655337 & 1.04953 \\
\hline
\end{tabular}
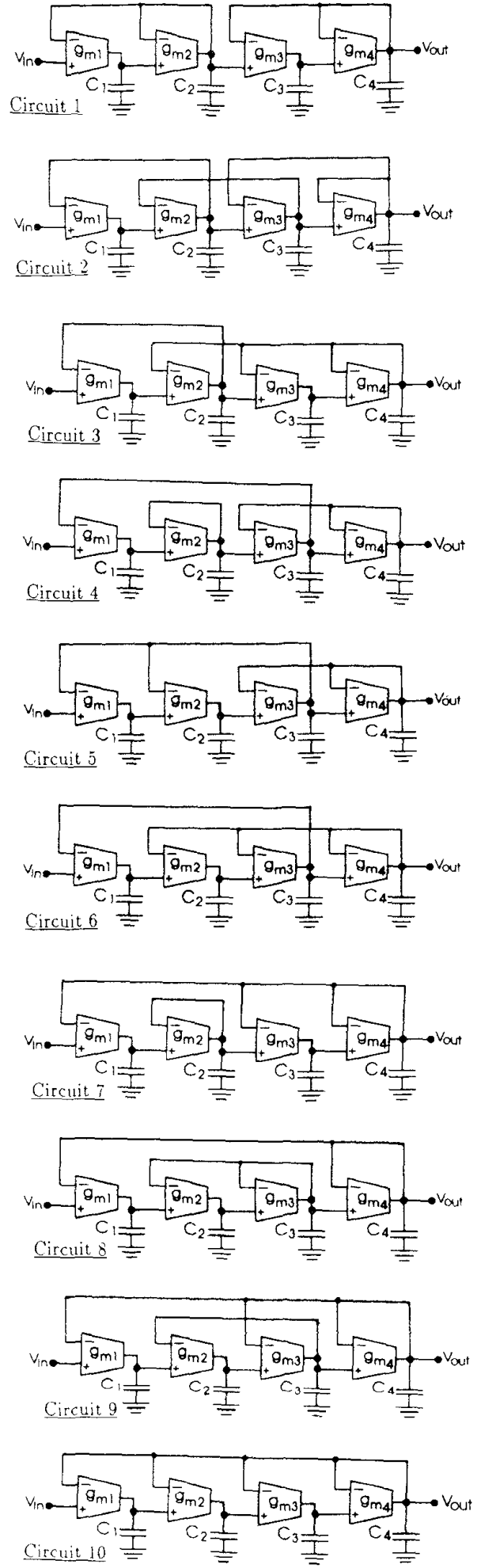

Figure 4: Fourth-order all-pole OTA-C filter structures 